山्山FARANÇAISE

$\gg \mathrm{DE}$

필 PEAGOGIE
Revue française de pédagogie

Recherches en éducation

182 | 2013

Connaissances et politiques d'éducation : quelles interactions?

\title{
Agulhon Catherine, Convert Bernard, Gugenheim Francis \& Jakubowski Sébastien. La professionnalisation. Pour une université « utile »?; Maillard Fabienne (dir.). Former, certifier, insérer. Effets et paradoxes de l'injonction à la professionnalisation.
}

Paris : L'Harmattan, 2012, 270 p. ; Rennes : Presses universitaires de Rennes, 2012, 254 p.

Cédric Hugrée

\section{(2) OpenEdition}

Édition électronique

URL : http://journals.openedition.org/rfp/4035

DOI : $10.4000 / \mathrm{rfp} .4035$

ISSN : 2105-2913

Éditeur

ENS Éditions

Édition imprimée

Date de publication : 28 août 2013

Pagination : 141-145

ISSN : 0556-7807

\section{Référence électronique}

Cédric Hugrée, « Agulhon Catherine, Convert Bernard, Gugenheim Francis \& Jakubowski Sébastien. La professionnalisation. Pour une université " utile » ?; Maillard Fabienne (dir.). Former, certifier, insérer. Effets et paradoxes de l'injonction à la professionnalisation. », Revue française de pédagogie [En ligne], 182 | 2013, mis en ligne le 28 août 2013, consulté le 22 septembre 2020. URL : http:// journals.openedition.org/rfp/4035; DOI : https://doi.org/10.4000/rfp.4035 
AGULHON Catherine, CONVERT Bernard, GUGENHEIM Francis \& JAKUBOWSKI Sébastien. La professionnalisation. Pour une université « utile» ? Paris : L'Harmattan, 2012, $270 \mathrm{p}$.

MAILLARD Fabienne (dir.). Former, certifier, insérer. Effets et paradoxes de l'injonction à la professionnalisation. Rennes : Presses universitaires de Rennes, 2012, 254 p.

\section{LES UNIVERSITÉS, L'EMPLOI, LA CRISE}

Dix années après l'obligation d'inscription des diplômes, notamment universitaires, au répertoire national des certifications professionnelles ${ }^{1}$ (RNCP) et cinq années après l'adoption de la loi du 10 août 2007 relative aux libertés et responsabilités des universités ${ }^{2}$ qui confère au service public d'enseignement supérieur une mission d'orientation et d'insertion professionnelle, deux ouvrages proposent plusieurs éléments d'interprétation de cette transformation récente qui déstabilise actuellement les universités françaises : l'accroissement, inédit d'un point de vue historique, des exigences de professionnalisation explicites de leurs enseignements, de leurs diplômes et finalement de l'ensemble de leurs filières.

L'ouvrage dirigé par F. Maillard n'accorde en fait que deux chapitres (écrits par C. Agulhon et J. Rose) à la professionnalisation dans les universités. La perspective transversale de l'ouvrage couvrant l'ensemble du système éducatif offre ici une occasion rare de mettre en perspective un processus dont l'analyse est trop souvent restreinte à l'enseignement professionnel. L'ouvrage co-écrit par C. Agulhon, B. Convert, F. Gugenheim et S. Jakubowski est quant à lui entièrement consacré à la professionnalisation à l'université. C'est ici la dimension socio-historique et monographique des deux recherches engagées dans les universités des académies de Lille et de Rennes qui permet d'apprécier les changements réels du dernier mouvement de professionnalisation des études universitaires au regard des formes variées qu'avait prises, par le passé, le rapprochement entre les diplômes universitaires et certains emplois qualifiés du secteur privé.

\section{Une autre professionnalisation est-elle possible?}

Laissant la dénonciation des récents développements de la professionnalisation de l'université à d'autres, les auteurs de ces deux ouvrages se retrouvent d'abord dans le souci de ne jamais séparer les savoirs académiques de leurs utilités professionnelles avérées. Repérable au sous-titre en forme de (timide) plaidoyer pour la professionnalisation (Pour une université «utile »?), ce parti-pris est détaillé par B. Convert, F. Gugenheim et S. Jakubowski lorsqu'ils écrivent dès le premier paragraphe de leur partie qu' «il est dans la nature de l'université de construire de la professionnalité » (p. 17). Les auteurs fondent ici leur analyse sur une lecture (inédite en France) de la critique faite par J. Meyer des approches fonctionnalistes de l'université ; le système scolaire et universitaire participant pleinement pour cet auteur « à la construction même de cette structure de positions, en créant sans cesse de nouveaux rôles et de nouvelles compétences » (Meyer, 1977). Les trois auteurs sont en quelque sorte rejoints par J. Rose (Maillard, 2012, p. 77-94) qui ne reconnaît pas « de véritable clivage entre les formations générales et professionnelles mais plutôt des degrés et des formes variables de professionnalisation » (p. 90). Contre les injonctions bureaucratiques actuelles, l'auteur plaide pour une véritable appropriation universitaire de la professionnalisation fondée sur la justice sociale, un usage raisonné des enquêtes d'insertion locales et une politique d'accompagnement des transitions professionnelles des étudiants. C'est pour l'auteur à ces conditions que l'université pourra imposer « sa » professionnalisation entendue comme « une formation non étroitement spécialisée, destinée à préparer à l'ensemble de la vie active et pas seulement au premier emploi, garantissant la transversalité et la transférabilité des acquis, intégrant la formation à l'emploi et à la recherche d'emploi, développant les capacités d'adaptation combinant formation théorique et apprentissage en situation de travail » (p. 91).

L'ensemble des auteurs des deux ouvrages insiste d'ailleurs sur l'ancrage historique de la volonté politique de mieux adapter l'offre des études universitaires aux besoins économiques. Néanmoins, ses développements récents n'ont, pour Fabienne Maillard, rien d'un « aménagement de l'offre des diplômes »; ils constituent au contraire une véritable « restructuration du système d'enseignement » en rupture avec le modèle universitaire (p. 241). Les inflexions les plus récentes de la politique universitaire sont rapprochées, dans 
l'ouvrage co-écrit par C. Agulhon, B. Convert, F. Gugenheim et al., d'une généralisation du "néomanagérialisme » qui transforme radicalement « l'appareil d'État » et ses institutions (p. 10).

La première partie de cet ouvrage (rédigée par B. Convert, F. Gugenheim et S. Jakubowski) interroge la professionnalisation dans les universités de l'académie de Lille à travers l'analyse monographique des deux principaux types de diplômes professionnalisants : 16 licences professionnelles et 26 masters professionnels, successeurs des diplômes d'études supérieures spécialisées (DESS) ${ }^{3}$. Cette partie, très riche empiriquement, fait également référence aux recherches réalisées sur ces questions aux États-Unis. La mobilisation du travail de R. E. Roemer (1981) permet aux trois auteurs d'avancer l'idée qu'en « matière de professionnalisation donc, pour des raisons historiques, les grandes écoles constituent en France le modèle dominant. Leurs diplômes sont autant de labels d'excellence à plus ou moins forte valeur symbolique, renforcée par le capital symbolique que constitue le réseau des anciens. [...] Autrement dit, en matière de professionnalisation, l'Université en France est dans une position de challenger par rapport aux écoles » (p. 20). Les conséquences de cette domination sont perceptibles dans l'imposition du niveau d'études bac +5 pour accéder au niveau de l'encadrement supérieur des entreprises privées et même dans plusieurs segments de la fonction publique. Elles sont aussi repérables dans la prégnance du modèle des écoles («autonomes » et « sélectives ») pour l'organisation de nombreuses filières professionnelles de l'université (p. 21).

\section{La professionnalisation d'avant la professionnali- sation (années 1960-1970)}

L'analyse socio-historique des différentes étapes de la professionnalisation dans les universités du Nord révèle la centralité de l'histoire économique locale dans le développement d'une offre universitaire professionnalisée. Si le terme ne fait son apparition qu'à l'occasion des Assises de l'enseignement supérieur de 1990, il existe bel et bien une «professionnalisation avant la professionnalisation » (p. 25) dans plusieurs spécialités universitaires telles que le droit et la santé (université de Lille 2). Plusieurs initiatives de professionnalisation sont également repérables dans d'autres composantes (tels le sport et la gestion), mais c'est au sein de la faculté de sciences que les auteurs repèrent un modèle particulier de professionnalisation : "Ce qu'il est important de noter à propos de ces créations, c'est qu'elles ne constituent pas, sauf exception, une réponse à la "demande" des industries locales. [...] Le vecteur de l'innovation va de l'université vers le monde industriel » (p. 30-31).
L'objectivation des parcours des enseignants porteurs de cette première professionnalisation révèle une génération de jeunes assistants et maîtres-assistants nés autour de 1940 qui a bénéficié des créations de postes des années 1960, génération au recrutement social plus populaire que la génération précédente, plus souvent passée par les Instituts de préparation à l'enseignement secondaire (IPES $)^{4}$ et membres du Snesup en 1968. La faculté des sciences est d'ailleurs à cette époque le lieu d'un débat vif sur le sens à donner aux formations entre les différents « mandarins patrons de labos » (p. 35) refusant toute perspective d'application et ceux souhaitant que leurs enseignements soient plus appliqués aux besoins de l'industrie. Les auteurs soulignent alors que le débat est « essentiellement disciplinaire, entre mathématiciens et physiciens d'une part, et représentants des sciences appliquées, électroniques et automatiques de l'autre ; mais il recouvre d'autres clivages de trajectoires (normaliens/ingénieurs) et idéologique (droite/gauche) ». Les positions des «professionnels» (par opposition aux " académiques »; p. 36) se comprennent aussi à la lumière de leur souci d'ouvrir "l'université à un éventail plus large de bacheliers [et de] "diversifier les filières de formation" jusque-là tournées, dans leur orientation principale, vers l'enseignement et la recherche » (p. 37). Et les auteurs de conclure qu'à Lille « ces mesures de professionnalisation avant la lettre ont donc ceci de paradoxal qu'elles sont menées par des hommes (beaucoup plus rarement des femmes) "de gauche", contestataires du pouvoir "mandarinal" en 1968 ». Si ces transformations pédagogiques sont loin de faire consensus parmi les disciplines scientifiques et les militants syndicaux, les auteurs font malgré tout l'hypothèse d'une division du travail universitaire entre les présidences d'universités qui prennent en charge ces transformations et les laboratoires soucieux de préserver leur politique de recherche (p. 52) dans un contexte de progression des effectifs étudiants.

Ce retour sur la première " explosion universitaire » abordée depuis la problématique de la professionnalisation est éclairant pour l'objet étudié : il autorise notamment en conclusion une comparaison de l'esprit des lois Faure et Pécresse en matière d'orientation et d'insertion (p. 55-56) et les paradoxes contemporains qui en découlent notamment à l'échelle des carrières des enseignants-chercheurs (p. 7780). Mais cette première monographie peut aussi être lue comme une contribution à l'histoire sociale des institutions académiques françaises, encore trop peu développée pour le cas de l'université française (Hugrée \& Soulié, 2013).

\section{Les licences et les masters professionnels : une professionnalisation " dés-universitarisée "?}

Dans la suite de cette première partie, les trois auteurs construisent une typologie des logiques de création 
des licences professionnelles dans l'académie de Lille. Ils identifient 5 types de licences allant de la licence professionnelle résultant d'une commande d'un milieu professionnel jusqu'à la licence proposant une articulation fine entre enseignement, recherche et milieu professionnel. Le modèle d'une licence professionnelle créant un nouveau domaine de professionnalité est ici envisagé comme un ultime idéal-type de professionnalisation, nettement mis en avant par les responsables institutionnels, dont les auteurs n'ont pourtant pas trouvé de traductions concrètes dans les universités enquêtées.

L'analyse spécifique des masters professionnels permet aux auteurs de reconstruire deux moments de la professionnalisation des diplômes universitaires. Les masters professionnels sont d'abord les héritiers des DESS, créés entre 1980 et 1995 et qui étaient souvent commandés par « le projet intellectuel et de carrière d'un enseignant» (p. 109). Pour les auteurs, ces diplômes de troisième cycle constituaient des formules originales de professionnalisation, à la fois distants des modèles des grandes écoles et informés par la recherche (p. 122). À l'inverse, les masters professionnels postérieurs à la réforme Licence Master Doctorat de 2002 $(\mathrm{LMD})^{5}$ sont commandés par des logiques d'institutions, de positionnement d'un établissement ou d'une filière dans un espace concurrentiel, ce qui contribue à affaiblir le rôle des projets intellectuels qui avaient fait les grandes heures de plusieurs DESS des universités observées. Dans cette optique, on comprend que l'effet du LMD est d'imposer une nouvelle logique de professionnalisation qui s'avère de plus en plus éloignée de l'utilité sociale et professionnelle des savoirs disciplinaires et académiques.

La «tourmente » (p. 126) dans laquelle se trouvent les acteurs de la professionnalisation semble encore plus notable dans l'académie de Rennes, où le développement de la professionnalisation s'est d'abord fait dans des disciplines dominées de l'espace académique local. La monographie proposée par C. Agulhon s'est d'abord heurtée à des difficultés d'accès au terrain. L'auteur mentionne ainsi « une vingtaine de rendez-vous pour une centaine de demandes par mail » (p. 140). Elle interprète ces refus répétés comme le « reflet des tensions qui travaillent l'enseignement supérieur» (p. 140). C'est sans nul doute un élément important de la situation universitaire bretonne, mais on pourrait aussi faire l'hypothèse que ces refus n'auraient pas été aussi nombreux si l'enquête avait été réalisée par un chercheur appartenant depuis plusieurs années à l'une des universités bretonnes. L'autochtonie et l'interconnaissance sont en effet des ressources décisives pour les enquêtes sur les institutions académiques ; elles sont des conditions-clés pour rencontrer les dirigeants et avoir accès aux archives administratives ainsi que j'ai pu en être témoin lors de ma propre enquête sur la professionnalisation de l'offre universitaire à Nantes.
Malgré tout, C. Agulhon retrace les grandes lignes de la transformation de l'offre de formation postérieure au LMD en Bretagne. La typologie présentée tout au long de cette seconde partie de l'ouvrage distingue les masters professionnels pionniers (créés avant le LMD), les masters créés dans l'urgence de la réforme LMD, les filières constitutives (département, voire discipline), les diplômes marginaux, les licences professionnelles universitaires « cherchant à concilier savoirs académiques et professionnels » (p. 198) et enfin les licences professionnelles pragmatiques, le plus souvent créées en IUT. Cette typologie rassemble donc plusieurs niveaux d'organisation institutionnelle (diplôme, discipline, filière) et se fonde sur plusieurs variables, parmi lesquelles on trouve l'origine du projet, l'intensité de l'implication du monde professionnel, la part de l'enseignement disciplinaire ou encore la part des financements propres (p. 241).

On ne comprend pas pourquoi l'auteur ne recourt pas à la typologie construite par ses co-auteurs dans la première partie de l'ouvrage. Le choix de C. Agulhon de placer sur un même plan à l'intérieur d'une même typologie les diplômes, les filières et les disciplines n'est pourtant pas sans poser question. La distinction établie par ses co-auteurs entre les licences professionnelles et les masters professionnels qui aboutit à une typologie séparée des licences professionnelles et des masters professionnels dans la première partie n'est l'objet d'aucun commentaire ; cela s'avère dommageable à l'entreprise de clarification initialement entreprise par l'auteur. Est-ce parce que cette première typologie apparaît peu adaptée à l'auteur pour penser le cas breton ? Dès lors, à quelles conditions est-il possible de généraliser les conclusions issues de ces deux monographies universitaires ? Par ailleurs, l'annexe (p. 241-243) et les pages 143 à 147 qui présentent les variables fondant cette nouvelle typologie ne disent rien de la manière dont ont été renseignées, construites et codées les caractéristiques institutionnelles des formations bretonnes. Ces variables n'ont pour la plupart rien d'une évidence et le codage des caractéristiques des formations observées repose sur un travail interprétatif qui est la plupart du temps passé sous silence, ce qui fragilise un peu plus la typologie présentée. La reprise de cette recherche dans l'ouvrage dirigé par F. Maillard (p. 63-76) pose d'ailleurs les mêmes questions.

\section{Le " retour de l'adéquationnisme " et ses effets sur l'espace de l'enseignement supérieur}

Ces deux ouvrages documentent donc dans deux perspectives complémentaires (monographique et comparatiste) ce " retour remarquable de "l'adéquationnisme" » (Maillard, p. 15), c'est-à-dire une vision des relations formationsemplois qui nie l'autonomie relative du système éducatif et cherche, en vain et malgré « la succession des preuves de son 
invalidité » (Maillard, p. 15), à établir une correspondance stricte entre ces deux espaces sociaux très segmentés. À ce titre, ces recherches sur la professionnalisation à l'université autorisent, à mon sens, deux prolongements en matière de recherche.

Le premier concerne les transformations du droit des diplômes et des certifications et ses impacts récents sur l'espace de l'enseignement supérieur. L'absence d'analyse de la professionnalisation en termes juridiques est un des regrets qui accompagne la lecture de La professionnalisation. Pour une université " utile »? L'histoire du droit des diplômes de l'enseignement supérieur s'avère pourtant riche d'enseignement tant la question des finalités professionnelles est intrinsèque à la création des grades et diplômes et au monopole de leur collation instauré dès 1808 (Caillaud, 2012, p. 45). Et ce n'est d'ailleurs pas la moindre conséquence de l'adoption de la réforme LMD que d'avoir cassé un des derniers monopoles juridiques des universités françaises : «[...] l'essentiel de la réforme du LMD a conduit l'État a créer un nouveau grade, le master, et à habiliter les établissements privés à le délivrer [...]. Du point de vue de la politique universitaire, le changement décisif vient du fait que désormais les établissements du secteur sélectif de l'enseignement supérieur, autres que les universités stricto sensu, ont obtenu le droit de délivrer des diplômes et des grades qui étaient jadis réservés à celles-ci » (Beaud, Caillé, Encrenaz et al., 2010, p. 61). L'intégration de la perspective juridique à l'enquête sociologique sur un tel objet s'avère heuristique en ceci qu'elle permet par exemple de traiter les arrêtés d'habilitation de diplômes à la fois comme des archives administratives objectivant les rapports en actes entre l'administration centrale et une université pluridisciplinaire en matière de construction d'une offre d'études, mais aussi de quantifier et caractériser les transformations de l'offre des diplômes d'une université. C'est cette perspective que j'avais pour ma part privilégiée afin d'analyser la progressive normalisation des diplômes professionnels au sein de l'université de Nantes entre 1980 et 2002 (Hugrée, 2010, 2012). Il faut enfin rappeler combien l'absence de construction socio-juridique du diplôme dans les analyses des diplômes de l'enseignement supérieur contribue à oublier que leur capacité et leur qualité « insertionnelles » dépendent largement de leur reconnaissance comme qualification dans les conventions collectives nationales de branches ou dans les trois fonctions publiques.

Le deuxième prolongement qu'ouvrent ces deux recherches renvoie à l'accès scolairement et socialement différencié des étudiants à ces formations, le plus souvent annoncées comme sélectives «sur le papier ». Le fait est évidemment mentionné par C. Agulhon, B. Convert, F. Gugenheim et alii (par exemple p. $122-123$ ou p. 214), mais il reste à décrire et à analyser les conditions réelles de cette soi-disant « sélection » des étudiants, dont on peut sans grand risque présager qu'elle varie très fortement selon à la fois la position de la formation au sein de l'espace universitaire national et local mais peut-être aussi en fonction des relations de cette formation avec certains segments du marché du travail. La déconstruction du label sélectif de ces formations apparaît en effet d'autant plus nécessaire que ces formations tendent aujourd'hui à s'imposer comme les modèles d'une université refondée. En un mot, il s'agit de comprendre les modalités par lesquelles ce « retour de l'adéquationnisme » favorise, dans le contexte universitaire et économique actuel, le « retour du sélectionnisme » (Neyrat, 2006).

Cédric Hugrée
Cresppa-CSU (CNRS/Université Paris 8-Vincennes-Saint-Denis)

\section{NOTES}

1 Loi $\mathrm{n}^{\circ}$ 2002-73 du 17 janvier 2002 de modernisation sociale, Journal officiel du 18 janvier 2002, p. 1008.

2 Loi $n^{\circ} 2007-1199$ du 10 août 2007 relative aux libertés et responsabilités des universités, Journal officiel du 11 août 2007, p. 13468

3 Diplôme créé par l'arrêté du 16 avril 1974, Journal officiel du 02 mai 1974, p. 4672

4 « Créés en 1957, ces instituts permettaient à des étudiants sélectionnés grâce à leur bons résultats en propédeutique d'être rémunérés durant leurs études jusqu'à la préparation du CAPES, puis plus tard de l'agrégation, sous réserve de s'engager à travailler 10 ans dans l'Éducation nationale " (note de bas de page $n^{\circ} 25$, p. 31).

5 Décret $n^{\circ} 2002-482$ du 8 avril 2002 portant application au système français d'enseignement supérieur de la construction de l'Espace européen de l'enseignement supérieur.

\section{BIBLIOGRAPHIE}

BEAUD O., CAILLÉ A., ENCRENAZ P., GAUCHET M. \& VATIN F. (2010). Refonder l'université. Pourquoi l'enseignement supérieur reste à reconstruire. Paris : La Découverte.

CAILLAUD P. (2012). " Les diplômes universitaires de l'enseignement supérieur : des certifications nationales/ générales ou professionnelles/locales ». In E. Quenson \& S. Coursaget (dir.), La professionnalisation de l'enseignement supérieur. Toulouse : Octarès, p. 35-46.

HUGRÉE C. \& SOULIÉ C. (2013) « De l'intérêt d'une histoire sociale des institutions académiques françaises avant 1968 ». Introduction à la première journée d'étude 50 ans après les Héritiers : le contexte académique, disciplinaire et intellectuel (années 1950-1964), organisée à Paris le 28 mars 2013.

HUGRÉE C. (2012). " L'insertion : nouvel enjeu local et national des politiques de l'enseignement supérieur? ". In V. Becquet, P. Loncle \& C. Van De Velde (dir.), Politiques de la jeunesse : le grand malentendu. Nîmes: Champ social, p. 138-156.

HUGRÉE C. (2010). L'échappée belle. Parcours scolaires et cheminements professionnels des étudiants d'origine 
populaire diplômés de l'université (1970-2010). Thèse de doctorat, sociologie, université de Nantes.

MEYER J. (1977). « The Effect of Education as an Institution ». American Journal of Sociology, vol. 83, no 1, p. 55-77.

NEYRAT F. (2006). " Le retour du sélectionnisme ». Les temps modernes, $\mathrm{n}^{\circ}$ 637-638-639, p. 364-392.

ROEMER R. E. (1981). «Vocationalism in Higher Education Explanations from Social Theory ". The Review of Higher Education, vol. 4, no 2, p. 23-46.

ANDRÉ Géraldine. L'orientation scolaire. Héritages sociaux et jugements professoraux. Paris : PUF, 2012, $172 \mathrm{p}$.

Publié en janvier 2012, l'ouvrage de Géraldine André repose sur une enquête ethnographique conduite entre 2005 et 2007 au sein de familles de classes populaires et de trois établissements scolaires d'une région belge en voie de désindustrialisation (Charleroi). Il présente deux principaux intérêts. Tout d'abord, celui de placer au cœur de l'analyse du processus d'orientation les héritages sociaux et familiaux des élèves. Ensuite, en articulant les apports des travaux de Pierre Bourdieu et de Paul Willis, l'ouvrage contribue au renouvellement de la sociologie de l'orientation scolaire, à partir de l'analyse des différentes formes de résistance culturelle des jeunes vis-à-vis de l'école.

\section{Formes de résistance " culturelle" face aux verdicts scolaires}

Les représentations liées à l'orientation des filles et des garçons des classes populaires vont dans le sens d'un choix subi, contrarié ou encore d'un vécu de relégation. Or, Géraldine André montre de manière très convaincante que les élèves se situent bien loin de ces images d'individus passifs, étrangères aux processus identificatoires qui se jouent dans la transmission de l'héritage familial. Son analyse documente l'ambivalence des enfants d'ouvriers envers les études. Ce constat s'inscrit dans la lignée des travaux de Paul Willis qui ont montré les processus par lesquels les enfants d'ouvriers (les «gars ») deviennent ouvriers : valorisation du travail manuel par rapport au travail intellectuel, valorisation de la force physique et des formes de virilité associées aux cultures d'atelier (Willis, 1978). L'analyse de Géraldine André s'ancre néanmoins dans un autre contexte socio-économique, celui d'une région en voie de désindustrialisation. Dans quelle mesure la dévalorisation du monde ouvrier qui en résulte (Mauger, 2006) n'est-elle pas un obstacle pour le développement d'une contre-culture scolaire à l'instar de celle des enfants d'ouvriers décrite par Paul Willis ? Dans quelle mesure sommes-nous en présence de la constitution d'un « nous » unificateur ? Géraldine André affirme la permanence d'une culture contre l'école, tout en nuançant toutefois ce constat en notant l'émergence de processus d'individuation et d'individualisation (« la tendance culturelle majoritaire dans le cadre de l'institution scolaire qui a été observée [...] est moins à la résistance qu'à la composition avec la culture scolaire », p. 162).

La première partie de l'ouvrage, par le détail des quinze portraits qui constituent son principal matériau d'analyse, montre comment les jeunes enquêtés entrent dans des processus d' « appropriation » puis de « réappropriation » (p. 87) de leurs parcours scolaires, dans le cadre de leur socialisation familiale puis de « cadres culturels déviants ou souterrains » (p. 96). Cinq formes de rapports à l'école sont ainsi identifiées, illustrées par des portraits de jeunes : la résistance, l'opposition, la culture comme principe, la dissociation conformiste et l'instrumentalisme.

Les concepts-clés de culture et d'identité de classes tendent cependant à figer des catégories qui, si elles s'inscrivaient dans une analyse davantage processuelle, pourraient éclairer le rôle des pairs, des rapports sociaux de sexe et des interactions avec les acteurs de l'institution dans les parcours scolaires des jeunes. On peut regretter que ces dernières dimensions, qui participent largement au processus d'orientation, soient insuffisamment mises en lumière par le découpage en deux parties distinctes de l'ouvrage. Celui-ci s'intéresse en effet dans un premier temps aux jeunes et à leur famille et dans un deuxième temps au rôle des acteurs institutionnels, et ne permet donc pas d'analyser les effets des jugements et des pratiques des agents institutionnels sur les enquêtés présentés en première partie. En effet, la focale est placée sur la socialisation familiale et les transmissions intergénérationnelles. Les rapports de domination au cœur des processus d'orientation relèvent aussi de processus de différenciation et de hiérarchisation sociales entre les hommes et les femmes, qui sont peu pris en compte ici : l'expression utilisée, au masculin neutre, de « jeunes de classes populaires » pour désigner les filles et les garçons, en témoigne.

\section{Stratégies de reproduction des établissements}

Un des apports importants de cet ouvrage est celui de traiter des stratégies de reproduction des établissements dans la hiérarchie scolaire locale. La seconde partie a pour objet « la manière par laquelle les acteurs institutionnels produisent de l'orientation scolaire en engageant et en produisant des significations, en contestant et en imposant du sens » (p. 99). Les enseignants enquêtés partagent un idéal commun, celui de «l'enseignement général pour tous » (p. 101), et des logiques de jugements liées à la « réalisation de soi », au « développement durable », au « projet », au 